\title{
TRABAJO Y EDUCACIÓN EN LA SEGURIDAD SOCIETAL
}

\author{
LUIS HOLDER \\ Universidad Simón Bolívar. Vicerrectorado Académico. ORCID: 0000-0001-6208-9272. \\ E-mails: luisholder@usb.ve; luisholder@hotmail.com

\section{VÍCTOR THEOKTISTO} \\ Universidad Simón Bolívar. Departamento de Computación y Tecnologias de la Información. \\ ORCID: 0000-0002-8045-4197.E-mail: vtheok@usb.ve
}




\section{TRABAJO Y EDUCACIÓN EN LA SEGURIDAD SOCIETAL}

La interrelación entre trabajo, educación y seguridad societal es un objeto de estudio e investigación con novedad original, que es investigado bajo el enfoque heurístico del modelo emergente de cuádruple hélice para la innovación en el dominio del ciberespacio; teniendo como actores a la sociedad civil, a los pueblos con su poder creador, a las instituciones de educación universitaria, a los gobiernos o administraciones de los Estados-nación, y a los sectores de la economía real y de la economía especulativa globalizante. Este artículo es un reporte preliminar de investigación, que presenta el estado del arte del trabajo y la educación como procesos categoriales que, objetiva o subjetivamente, impactan a la seguridad societal, y tienen efectos en la seguridad de los Estados-nación y la Paz internacional.

Palabras Chave: Seguridad Societal. Educación. Trabajo. Cuádruple Hélice. Paz. Ciberespacio.

\section{LABOUR AND EDUCATION IN SOCIAL SECURITY}

The relationships among education, labour and societal security is an object of study and research with original novelty, which has been investigated under the heuristic approach of the emerging quadruple helix model for innovation in the cyberspace domain; having as actors the civil society, the people with their creative power, the institutions of university education, the governments or administrations of the nation-states, and the sectors of the real economy and the globalizing speculative economy. This article is a preliminary research report, publishing the state of the art for labour and education as categorical processes that objectively or subjectively impact on societal security, and has effects on the security of nation-states and international peace.

Keywords: Societal Security. Education. Labour. Quadruple Helix. Peace. Cyberspace.

\section{TRABALHO E EDUCAÇÃO EM SEGURIDADE SOCIAL}

A inter-relação entre trabalho, educação e seguridade social é um objeto de estudo e pesquisa original, investigada sob a abordagem heurística emergente do modelo de hélice quádrupla para inovação no domínio do ciberespaço; tendo como atores a sociedade civil, os povos com seu poder criativo, as instituições de ensino universitário, os governos ou administrações dos Estados-nação e os setores da economia real e da economia especulativa globalizada. Este artigo é um relatório de pesquisa preliminar, que apresenta o estado da arte do trabalho e da educação como processos categóricos que impactam, objetiva ou subjetivamente, a seguridade social, e têm efeitos na segurança dos Estados-nação e na paz internacional.

Palavras Chave: Seguridade Social. Educação. Trabalho. Quadruplo Hélice. Paz. Ciberespaço. 


\section{TRABAJO Y EDUCACIÓN EN LA SEGURIDAD SOCIETAL}

\section{Introducción}

A mediados de la década de los años ochenta del siglo XX se funda el Copenhague Peace Research Institute (COPRI), como institución de investigación multidisciplinaria en las disciplinas de Paz y Seguridad, adscrito al Parlamento de Dinamarca. En Enero de 2003, COPRI fue transformado en el Instituto Danés de Estudios Internacionales. Barry Buzan, académico fundador del COPRI y Profesor Emérito de la Universidad de Copenhague, con una metodología y enfoque emergente se planteó la hipótesis de que la seguridad en el marco de la teoría de las relaciones internacionales, trasciende al Estado-nación. El hallazgo de investigación y lo que comprobó Buzan, fue que en adición a los ámbitos tradicionales como lo son el político, económico, militar y social; es posible identificar y debidamente caracterizar a otros dos ámbitos de singular significación, como lo son, el ambiental y el societal [BUZAN, 1983].

En este artículo, orientaremos la argumentación de los resultados de la investigación hacia el factor o ámbito societal y la seguridad asociada, es decir, el objeto de estudio central será la seguridad societal. Es conocido que la supervivencia del Estado-nación se correlaciona con la categoría soberanía, mientras que para el aspecto societal se preserva la identidad como esencial a la seguridad. En sentido de negación, una situación de inseguridad societal se da cuando un grupo social significativo ve su identidad y cultura amenazadas. "Cuando se habla de seguridad societal, se tocan aspectos relacionados con la identidad de un pueblo como lo son la lengua, la cultura, la religión o la nacionalidad. En otras palabras, cuando un pueblo tiene en riesgo su cultura, sus instituciones y estilo de vida, tiene también amenazada su seguridad." [GONZÁLEZ, 2016].

El vocablo societal fue asimilado al idioma castellano como un anglicismo. En la versión más reciente del diccionario Collins, la palabra Societal es un adjetivo que significa todo lo "relating to society or to the way society is organized" ("relacionado a la sociedad o al modo como la sociedad está organizada"), con dos acepciones que se vinculan con "societal changes" (cambios societales) y "societal norms" (normas societales). En el diccionario Oxford se amplían las acepciones a "complex structure" (estructura compleja), "cultural context" (contexto cultural) y "values" (valores). Niklas Luhmann [RODRÍGUEZ, 1990], sociólogo alemán, propuso una distinción entre sistemas sociales y sistemas societales: "Con el término societal se hace referencia a la sociedad. Un sistema societal es una sociedad, en tanto sistema de tipo propio. Un 
sistema social, en cambio, es un sistema que puede ser una sociedad, una organización o una interacción." Los que defienden el uso de societal en español o en francés, consideran que con este adjetivo se alude exclusivamente a la sociedad humana. Lo societal es un constructo que en esencia implica sociedad; sus estructuras, sistemas, instituciones y organizaciones. Incluye además, el accionar del ser humano, como "constructor social de la realidad". Actúa como un eje transversal para las diferentes disciplinas y ciencias de la sociedad. Aborda la evolución de los aportes del ser humano en sociedad, en los determinísticos tiempos históricos primitivos, tradicionales y en los modos de la modernidad o posmodernidad. En castellano existe el adjetivo societario: "Relativo a las asociaciones, especialmente a las obreras" [ESTECCHE DE FERNÁNDEZ, 2014], y también para las sociedades empresariales o mercantiles.

La "Seguridad Societal", se propone para tratar los nuevos problemas de los grupos sociales y conflictos al interno o con el entorno, los cuales tienen una identidad determinada y que ven amenazada, en riesgo, peligro o vulnerabilidad su existencia, como consecuencia de factores o agentes con intereses antagónicos entre grupos o comunidades que territorialmente pueden o no compartir recursos, pero le dan preeminencia a lo axiológico en los contextos cultural, social, ambiental y étnico; que superan aquellos principios y valores instituidos por los Estados-nación formalmente constituidos. En esa manera, surgirán nuevos problemas o conflictos que ameritan ampliar los enfoques, métodos y categorías de análisis. Con cierta similitud a la seguridad humana, aspectos de la seguridad societal remiten, por un lado, a la seguridad contra amenazas crónicas como el hambre, las enfermedades y la falta de libertades individuales; por otro, a la protección contra alteraciones súbitas y dolorosas del modus vivendi cotidiano en los planos familiar, laboral y/o comunitario; aunque las categorías más pertinentes sean seguridad económica, seguridad alimentaria, seguridad en materia de salud, seguridad ambiental, seguridad política y seguridad ciudadana.

El contexto para el estudio de la seguridad societal se sitúa en los ámbitos nacional e internacional, con la impronta de corrientes de pensamiento e ideologías que deben considerar la dinámica de los movimientos sociales y la globalización soportada en las nuevas formas de comunicación y procesamiento de información, al igual que en dominios de relaciones cooperativas o antagónicas como lo constituye en la actualidad el ciberespacio, que abre virtualmente fronteras, $\mathrm{y}$ en lo concreto crea un imaginario colectivo en los pueblos que incentiva el proceso de migraciones. Concomitante a las seguridades en lo nacional e internacional, la seguridad societal es impactada por las que conocemos como redes sociales y en consecuencia la ciberseguridad, será inexorablemente de interés para proveer tranquilidad a las comunidades virtuales. En otro orden de ideas, se reconoce la seguridad de los pueblos [SECRETARÍA DE SEGURIDAD MULTIDIMENSIONAL DE LA OEA, 2005], como se la asimiló en la Secretaría

\footnotetext{
p/Ulails Salvador, v. 4, n. 3, p. 09-35, set./dez. 2019
} 
de Seguridad Multidimensional de la Organización de Estados Americanos, considerando que por el principio de la soberanía popular, la seguridad de los pueblos es equiparable a la tradicional seguridad de los Estados, por lo cual esta última se debería armonizar y contribuir con la materialización objetiva de la seguridad de los pueblos, especialmente aquellos de naturaleza aborigen e indígenas de América Latina y el Caribe.

El nacionalismo y las presiones en la delimitación de las fronteras entre los territorios de los Estados, son causas de tensión entre los grupos de seres humanos, especialmente aquellos de diversos orígenes étnicos y nacionalidades. la seguridad societal también está estrechamente relacionada con la "seguridad social" del campo de las relaciones de trabajo o laborales; como producto de las amenazas desde los ámbitos sociopolíticos y socioeconómicos por discriminaciones, la delincuencia, las drogas y el terrorismo; o por vulnerabilidades como el analfabetismo, las enfermedades, la pobreza y salarios misérrimos o condiciones de esclavitud. La seguridad societal también puede verse amenazada por problemas internacionales o por las amenazas tradicionales de la seguridad nacional o de la Seguridad del Estado-nación. Si se aprecia un grado de inseguridad en lo societal de forma objetiva o subjetiva, procede entonces planificar contramedidas para mitigar amenazas o anular agresiones, para resarcir la identidad y valores culturales del colectivo o comunidad.

Los prolíficos aportes del COPRI, mejor conocida como la Escuela de Copenhague de Complejos Regionales de Seguridad, y en este siglo XXI se ha bautizado como la Escuela de Seguridad de Europa en virtud a que ya se considera parte de la doctrina de los Estados que se agrupan en la OTAN o NATO [MOLLER, 2000]; motivado quizás por la idiosincrasia especialmente de los países europeos ubicados en la región de Escandinavia y en el mar Báltico, en favor de la Paz Internacional, como históricamente lo han demostrado esas sociedades; adoptaron el paradigma de la seguridad societal. En ese sentido, desde comienzos de este siglo se ha estado fortaleciendo la cooperación multilateral de Noruega, Suecia, Dinamarca, Finlandia, Islandia, Estonia, Letonia, Lituania, Polonia, Rusia y Bielorrusia; en lo concerniente a acordar una agenda en la cual se tratasen los problemas que surjan en el seno de la seguridad nacional e internacional y que puedan ser analizados y resueltos, en la perspectiva y logro de los objetivos de seguridad societal.

Esos países citados en el párrafo anterior, crearon una bitácora con el registro de las lecciones aprendidas y las mejores prácticas de la agenda en materia de seguridad societal que se intitula, "Seguridad societal en la región del mar Báltico: identificación de expertos y relevancia en las políticas públicas" [AALTOLA, 2018], y en la cual se evalúa la evolución de la noción de seguridad societal en las narrativas y estrategias nacionales, los enfoques nacionales del Estado como proveedor de seguridad y el desarrollo del enfoque integral de la seguridad, en la región del 
mar Báltico. Cada vez es más relevante revisar la diversidad de enfoques nacionales de seguridad, ya que los desafíos contemporáneos y por ampliarse el espectro de seguridad, están surgiendo tanto dentro como fuera de la región, con potencial para impactar a nivel Europeo; y sistematizar aprendizajes en las mejores prácticas nórdicas, cuando se involucran a los actores sociales para garantizar la seguridad de esos Estados-nación en el plano interregional.

Las principales narrativas de seguridad societal varían en la región del mar Báltico, desde aquellos relacionados con identidades nacionales, tensiones interétnicas en los ambientes frágiles de los entornos cibernéticos, amenazas ecológicas, coyunturas de la economía, el sistema de bienestar socio-laboral, infraestructura y servicios, violencia de género; a conceptualizaciones más convencionales, relacionadas con defensa militar, protección civil y defensa psicológica. La diferencia más notable radica en los modelos altamente centralizados, como los de Bielorrusia y Rusia; a aquellos descentralizados y horizontales, como Suecia; y los modelos de co-gobierno, con una o más instancias de coordinación con la sociedad civil, como en los casos de Letonia y Estonia. Sin embargo, en la mayoría de los países señalados, el sector no gubernamental de empresas del sector agroalimentario y empresas relacionadas con infraestructuras críticas, tienen un compromiso cierto con la garantía y preservación de la seguridad societal.

En más de cuatro décadas contadas a partir del comienzo de la década de los años noventa en el pasado siglo XX, el constructo de seguridad societal generado por la Escuela de Copenhague de los Complejos Regionales de Seguridad, ha influenciado positivamente en las políticas públicas de la región báltica, con reconocimiento en la teoría y praxis concreta; y ya se considera como un paradigma consolidado, que se constituye en referente para otros enfoques de la seguridad a todos los niveles y ámbitos, a pesar de que algunos países generen también otras categorías de análisis y constructos plausibles, como seguridad de la patria en Estados Unidos, defensa total en Singapur, protección contra desastres y preparación para emergencias en Estados Unidos y seguridad humana en Japón. Venezuela ha venido desarrollando el paradigma de Defensa Integral de la Nación desde hacen aproximadamente seis décadas [MALDONADO MICHELENA, 1962].

En cuanto a la atención que ha tenido la seguridad societal en los espacios académicos, se observa que en países como como Letonia y Lituania [DE ESPONA, 10], han sido de interés para algunas universidades, grupos de investigadores expertos y centros de investigación, aun cuando lentamente se ha ido incorporando a las agendas gubernamentales de esos países. A título de ejemplo, hacemos referencia a una institución que desde 2011 ha está avanzando en líneas de investigación vinculadas a la seguridad societal en la Universidad de Gotemburgo en Suecia [Centro de Investigación de Protección Urbana y Seguridad Societal, 2011]; cuya misión es facilitar, coordinar y realizar investigaciones colaborativas que contribuyan a contrarrestar,

P/Uld $a$ i $S$ Salvador, v. 4, n. 3, p. 09-35, set./dez. 2019 
reducir y gestionar riesgos, amenazas, inseguridades y vulnerabilidades de manera que se construya una sociedad resiliente y socialmente inclusiva.

Asimismo, como parte del ecosistema de organizaciones no gubernamentales de pensamiento estratégico (Think Tank), está el conocido internacionalmente como VICESSE en Austria [CENTRO DE VIENA PARA LA SEGURIDAD SOCIETAL, 2015], dedicado a la investigación y consultoría, en una amplia gama de problemas de seguridad en un contexto societal e histórico, para identificar problemas de seguridad y proponer soluciones novedosas a nivel local, nacional y en la Unión europea. VICESSE es miembro promotor de la SOURCE, como red académica e intelectual de excelencia para vincular actores interesados en las dimensiones societales de la investigación, el desarrollo y la sistematización de la seguridad. SOURCE la integran entre otros miembros, las siguientes organizaciones: Norwegian Defence Research Establishment (FFI), Research Centre in International at Relations (RCIR) del King's College of London y Fraunhofer Group for Defence and Security (FRAUNHOFER-GESELLSCHAFT) en Alemania.

La metódica para el diseño de investigación de la seguridad societal como objeto de estudio de los autores de este artículo, se sustenta en la heurística, enfoque a través del cual es posible con libertad en lo ideológico, pensamiento crítico, pensamiento divergente y pensamiento estratégico, con las artes y las ciencias, aproximarnos a la formulación de constructos teóricos, y aplicaciones para resolver problemas o planificar y gestionar la seguridad societal, como disciplina que debe integrarse a las políticas de seguridad de los estados Nación, para coadyuvar con la Paz internacional, y los procesos igualmente societales de Educación y Trabajo [MESSINA, 2008].

\section{Seguridad societal y estándares}

Los estándares o normas técnicas son instrumentos normativos que regulan los procesos, sistemas, bienes y servicios de las economías en los ámbitos nacionales e internacionales. De alcance internacional, existen fundamentalmente una organización para los estándares técnicos [Organización Internacional de Normalización, 2019] y otra para la normalización de las telecomunicaciones [Unión Internacional de Telecomunicaciones, 2019], ambas con sus sedes en Ginebra. Esas dos instituciones han promulgado un conjunto de estándares, que son creados por comités de expertos en las diferentes áreas científico-técnicas y económicas, y que se constituyen en referentes para los Estados, que con independencia las adoptan o no, a través de sus órganos nacionales de normalización. 
Para el interés de esta investigación, la seguridad societal en la interdisciplinaridad y transdisciplinaridad, se ha evidenciado que en el contexto de la institucionalidad vinculada a estándares, se ha también conceptualizado y caracterizado en una perspectiva que es menester el considerar. La acepción de la seguridad societal en el contexto de los estándares técnicos, surge cuando se actualiza y sustituye el estándar británico BS25999-2 que se venía aplicando internacionalmente desde el año 2006, por uno que elaboró el comité ISO/TC 223 "Societal Security" de la ISO. La razón de haber asignado como área de responsabilidad la dimensión seguridad societal al comité ISO/TC 223, fue en virtud a que el cuerpo directivo de la ISO, consideró que lo societal era medular en los procesos superación de la interrupción en las operaciones de una empresa o institución, es decir, que no se debe soslayar la cohesión y los valores de identidad del grupo con la organización en la cual laboran y desarrollan un sentido de pertenencia. El principal aporte del ISO/TC 223 "Societal Security" fue la producción del cuerpo normativo que se vincula a los "Sistemas Gerenciales para la Continuidad de las empresas privadas [o instituciones del sector público"]:

ISO 22300:2012 Societal Security - Terminology

ISO 22301:2012 Societal security - Management systems - Requirements

ISO 22311:2012 Societal security - Interoperability

ISO 22313:2012 Societal security - Management systems - Guidance

ISO 22315:2014 Societal security - Mass evacuation - Guidelines

ISO 22317:2015 Societal security - Management systems - Guidelines

ISO 22320:2011 Societal security - Emergency management - Requirements

ISO 22322:2015 Societal security - Emergency management - Guidelines

ISO 22324:2015 Societal security - Emergency management - Guidelines

ISO 22397:2014 Societal security - Guidelines

ISO 22398:2013 Societal security - Guidelines

ISO/TR 22312:2011 Societal security - Technological capabilities 
ISO/TR 22351:2015 Societal security - Emergency management - Message structure

ISO/PAS 22399:2007 Societal security - Guideline for preparedness

El objetivo de los estándares de la serie ISO 223 es mantener una organización en funcionamiento durante y después de una interrupción, garantizando de esta forma que los productos y servicios serán entregados a los clientes puntualmente. Específicamente, el estándar ISO 22301:2012 proporciona todos los requerimientos necesarios para diseñar, implantar, Establecer, implantar, mantener y mejorar y certificar un Sistema de Gestión de Continuidad de las empresas [o instituciones del sector público]. La norma ISO 22301 incorpora lineamientos de nivel estratégico, operacional y táctico para la formulación y materialización del Sistema de Gestión de Continuidad de Negocio.

Es pertinente precisar que la aplicación del estándar está orientado al sector privado en la acepción del término "business", que se traduce como negocios o empresas; pero su alcance se amplia y adapta a cualquier tipo de organización privada o institución del Estado a nivel nacional, estatal, regional, comunal, municipal y local; al igual que para las pequeña y medianas empresas, o para grandes empresas nacionales o corporaciones transnacionales. ISO 22301:2012 es una norma internacional aplicable a todo tipo de organizaciones, públicas o privadas, independientemente de su tipo, tamaño o servicio prestado. Cobra especial relevancia en organizaciones que operan en entornos de alto riesgo, donde la necesidad de continuar las actividades laborales o trabajo, es de suma importancia para el negocio, clientes y la sociedad en general.

Las organizaciones independientemente de su propiedad, sea está del Estado o de naturaleza privada, a lo largo de sus vidas empresariales o institucionales, con una determinad probabilidad se encuentran sometidas a riesgos o incertidumbres, que podrían impactar su capacidad de crecimiento y supervivencia. Es por ello que se hace necesario que en las empresas e instituciones se elaboren e instauren los respectivos Sistemas Gerenciales de Continuidad de la Empresa o Institución, para anticiparse a las amenazas, peligros y vulnerabilidades que potencien los riesgos; con el propósito de mitigar o enfrentar la ocurrencia de una perturbaciones o daño que alteren las operaciones y funcionamiento permanente, de forma que se pueda garantizar el que se pueda continuar con la ejecución de las actividades cotidianas en un porcentaje aceptable de operatividad. 
Con los estándares de la serie ISO 223 es posible garantizar una efectiva planificación y programación de acciones para la continuidad de la empresa y la institución en los estamentos estratégico, operacional y táctico; permitiendo:

a.Focalizar los procesos críticos que impactan en la misión.

b.Fortalecer con métodos y tecnologías la capacidad de recuperación.

c.Elevar la resiliencia sobre otros actores que compiten en el entorno.

d.Posicionar imagen de confiabilidad ante clientes y comunidades en general.

e.Mejorar la imagen ante las empresas de seguros y reaseguros.

f.Estimar el impacto en la estructura de costos por incidentes e interrupciones.

g.Proyectar la esperanza de vida de la empresa o institución y de su personal.

h.Fortalecer la Higiene, el Ambiente y la Seguridad Laboral.

i.Restablecer en tiempos óptimos la continuidad con métodos científicotécnicos.

j.Educar para generar una cultura de seguridad societal corresponsable.

k.Estimar la aversión por el riesgo de la organización.

1.Establecer y comunicar la política de continuidad de negocio.

m.Asignar responsabilidades y niveles de autoridad para la seguridad societal.

n.Comprender la cultura organizacional y del entorno.

o.Consolidar la cohesión y el liderazgo adaptativo en la organización.

Las empresas o instituciones, que obtengan certificación ISO 22301:2012 de parte del órgano regulador del Estado con competencia en Normas Técnicas (Figura 1), tienen la conformidad de la metodología y las prácticas implantadas para mantener el negocio (industria o servicio) o institución pública funcionando a pesar de condiciones adversas, minimizando el impacto de potenciales incidentes en los clientes, la comunidad, partes interesadas y en general del ecosistema socioeconómico y societal. El certificarse en ISO 22301:2012 disminuye las vulnerabilidades en cuanto a seguridad societal, potencia la gobernanza de los entes, el de las leyes y estándares, proyectando la imagen y reputación empresarial o institucional, creando un 
clima de organizacional estable y de corresponsabilidad en relación al Estado, la sociedad, los trabajadores en general, proveedores, consumidores y clientes, y la ciudadanía en general.

Figura 1. Certificación ISO en Gerencia de la seguridad societal.

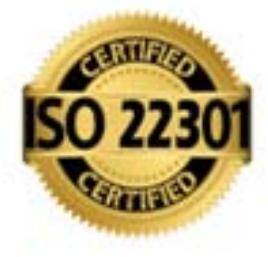

Fuente: Copyright $(0) 2015$ de ISO.

En 2014, la Junta de Gestión Técnica de ISO decidió fusionar ISO/TC 223 con otros comités en el área de seguridad para una mejor coordinación. El nuevo comité comenzó a funcionar desde el 1 de enero de 2015, con el nombre de COMITÉ ISO/TC 292 DE SEGURIDAD Y RESILIENCIA. En el segundo trimestre de este año 2019, el Comité ISO/TC 292 DE SEGURIDAD Y RESILIENCIA produjo el borrador del nuevo estándar ISO DIS 22301:2019 que intitulara "Security and resilience - Business continuity management systems - Requirements", para reemplazar al estándar ISO 22301:2012.

En síntesis, el Comité ISO/TC 223 fue el ente de la ISO que desde su conformación en 2001 y hasta su disolución en 2014, desarrolló los estándares internacionales que sentaron las bases de la gerencia científico-técnica de la seguridad societal, es decir, la administración de recursos públicos y privados para proteger a la los entes económicos e institucionales de la sociedad; y responder a incidentes, emergencias y desastres causados por actos humanos intencionales y no intencionales, riesgos naturales y fallas técnicas. El Comité ISO/TC 223 siempre visualizó y asumió a la seguridad societal como multidisciplinaria, transdisciplinaria e interdisciplinaria; que involucra a actores de los sectores público y privado, incluidas las organizaciones sin fines de lucro. 


\section{Seguridad societal y estándares}

En el estudio de los procesos de innovación en tecnologías, es de aceptación generalizada que al menos tres actores se interrelacionan sistémicamente. La universidad, industria y gobierno; desarrollando mayormente relaciones de cooperación y con el propósito del crecimiento equilibrado y armónico de cada uno de los tres sectores, es decir en sinergia entrópica, para conformarse en el que se conoce como modelo de Triple Hélice de [ETZKOWITZ, 1995]; el cual se ha constituido en un instrumento de la heurística para analizar los procesos de innovación y como elemento normativo para formular políticas de innovación. El modelo de Triple Hélice, aun cuando permite el potenciar la innovación, se critica porque epistemológicamente no incorpora las variables o categorías propias en la dimensión societal. La experiencia ha demostrado también que con la participación activa del gobierno como fiel de la balanza entre el Estado y la sociedad, se ha producido la acumulación de conocimiento científico y saberes del pueblo para tener aproximaciones de solución a determinados problemas en lo militar, social, económico y político.

En respuesta a esas limitaciones en lo societal, surge desde finales de la primera década del siglo XXI, una corriente de pensamiento de la sociedad del conocimiento y saberes de las comunidades en general; que incorpora un cuarto ente, la sociedad civil o pueblo con sus capacidades creadoras para innovar desde las ciencias, artes y oficios. El modelo de Cuádruple Hélice, integra a la industria, al gobierno del Estado-nación, a la Instituciones de Educación y a la sociedad civil. Para que se garantice la seguridad societal, es necesario que tanto el Estado-nación y la sociedad civil tengan intereses concurrentes, y que para el logro de sus fines de desarrollo integral y proyección histórica, se sustenten al menos en dos procesos fundamentales como lo son la educación y el trabajo, con conciencia e internalización del principio de corresponsabilidad entre el Esta-nación y la sociedad civil. 
Figura 2. Modelo de Cuádruple Hélice.

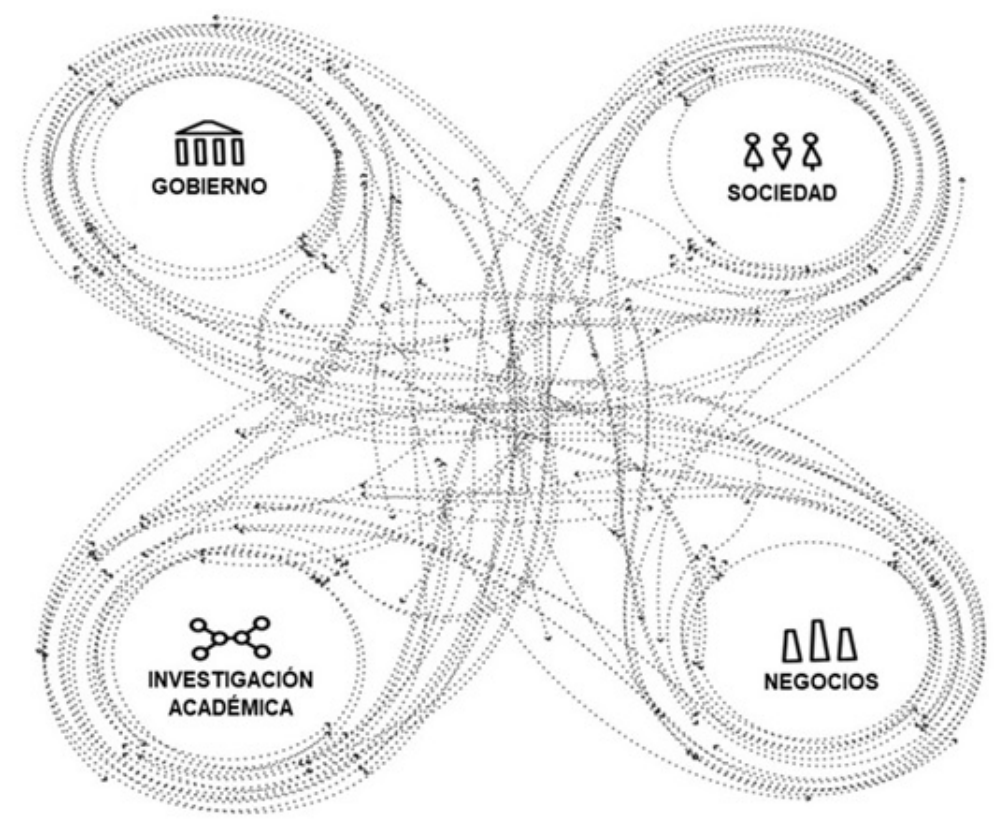

Fuente: Copyright $(0) 2015$ de FRAUNHOFER.

El sociograma de interrelaciones complejas como se ilustra en la Figura 2 [SCHÜTZ, 2019], que integra a Universidades (INVESTIGACIÓN ACADÉMICA), al Gobierno, a los sectores Industria y Comercio (NEGOCIOS), y a la Sociedad Civil (SOCIEDAD); en una óptica visual de la la hélice desde arriba, mostrando claramente que los cuatro componentes centrales de este sistema cuádruple de innovación se integran en interacciones bidireccionales, dinámicas y multicapas, destacando el papel de la sociedad como actor principal en los sistemas nacionales de innovación, así como la importancia de integrar activamente a la ciudadanía en los proyectos de innovación; en correspondencia con incorporar a la Sociedad Civil como ente también protagónico en los procesos de innovación [CARAYANNIS, 2009] con equilibrios entre las tendencias ideológicas más representativas en un mundo pluripolar y multicentrico [HOLDER, 2014]. Al tener visibilidad y participación protagónica la sociedad civil en el modelo de innovación, indirectamente también, se materializa el principio de corresponsabilidad en relación al tipo específico de Estado-nación (Figura 3). 
Figura 3. Significación de las ideologías en el modelo de Cuádruple Hélice.

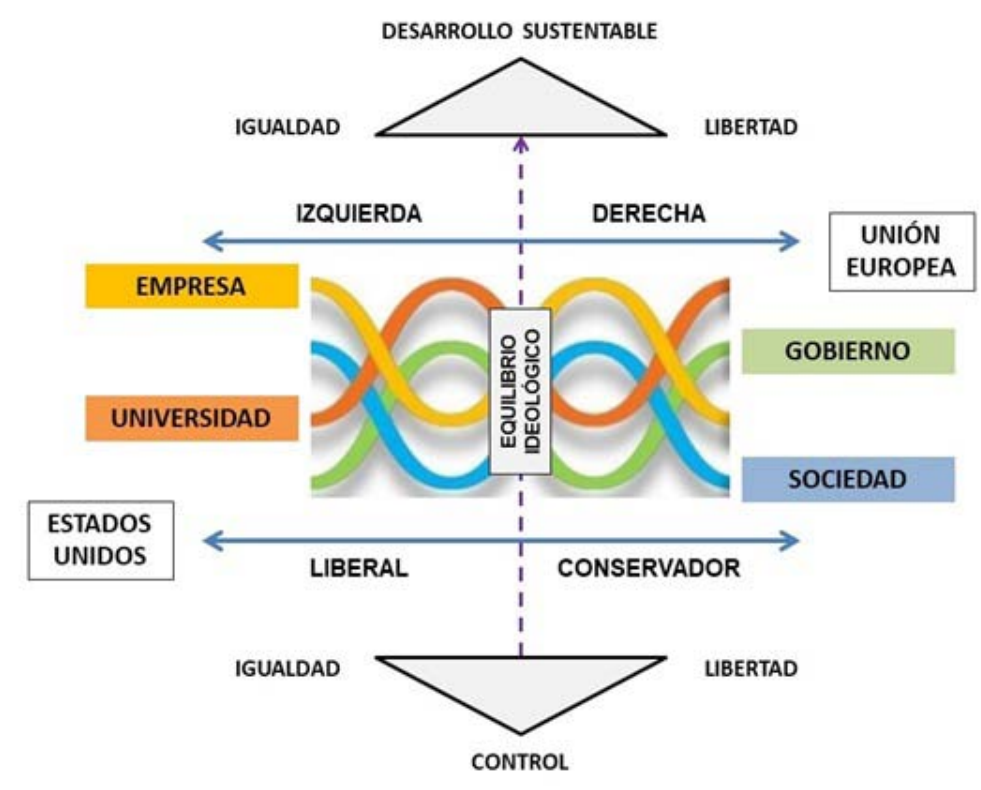

Fuente. Adaptación después de Carayannis y Campbell (2009).

\section{La seguridad de la Nación y el modelo societal de Cuádruple Hélice}

En 1999, el pueblo como ente en el cual reside la soberanía originaria, convocó una Asamblea Constituyente en Venezuela, con el propósito de refundar el Estado-nación y general una nueva Carta Magna. En la perspectiva teórica del Derecho Constitucional comparado, la Carta Magna de Venezuela, contiene una impronta axiología que consagra un conjunto de principios y valores que sustentan la seguridad societal [CONSTITUCIÓN DE LA REPÚBLICA BOLIVARIANA DE VENEZUELA, 1999]:

Artículo 1. Venezuela (...) fundamenta su patrimonio moral y sus valores de libertad, igualdad, justicia y paz internacional, en la doctrina de Simón Bolívar, el Libertador. (...). (el resaltado y subrayado es de los autores)

Artículo 2. Venezuela se constituye en un Estado democrático y social de Derecho y de Justicia, que propugna como valores superiores (...), la 
responsabilidad social y en general, la preeminencia de los derechos humanos, la ética y el pluralismo político. (el resaltado y subrayado es de los autores)

Artículo 3. El Estado tiene como fines esenciales la defensa y el desarrollo de la persona y el respeto a su dignidad (...). La educación y el trabajo son los procesos fundamentales para alcanzar dichos fines." (el resaltado y subrayado es de los autores)

Artículo 4. La República Bolivariana de Venezuela (...) se rige por los principios de integridad territorial, cooperación, solidaridad, concurrencia $\mathbf{y}$ corresponsabilidad. (el resaltado y subrayado es de los autores)

Artículo 326. La seguridad de la Nación se fundamenta en la corresponsabilidad entre el Estado y la sociedad civil (...). El principio de la corresponsabilidad se ejerce sobre los ámbitos económico, social, político, cultural, geográfico, ambiental y militar. (el resaltado y subrayado es de los autores).

A la luz del texto constitucional venezolano, se identifican a la Paz Internacional, la responsabilidad social, los derechos humanos, la concurrencia, la corresponsabilidad, la seguridad de la nación, la corresponsabilidad ente el Estado-nación y la sociedad civil, todas como categorías de análisis relevantes en la investigación; pero de gran significación es que para el logro de los fines del Estado democrático y social de Derecho y de Justicia; la educación y el trabajo son los procesos fundamentales para alcanzar dichos fines.

En síntesis, el modelo de Cuádruple Hélice se debe ubicar para su análisis y desarrollo de los procesos de innovación, en el paradigma venezolano de Seguridad de la nación, se vincula a través de los principios de corresponsabilidad y concurrencia con el Estado-nación en favor de un desarrollo armónico y simultaneo de la sociedad civil, sin soslayar que la soberanía reside intransferiblemente en el pueblo (Artículo 4 de la CRBV). En los estrictamente societal, este modelo que funciona bajo grados de libertad para la sociedad civil que a través de las redes sociales soportadas por el ciberespacio, podrá extender sus espacios públicos para la producción cooperativa en la llamada industria 4.0 que se ha estado consolidando con los FabLab, para la producir a escala industrial, artesanal y artística con altos grados de innovación en un modelo de desarrollo humano y social que sea sustentable e integral.

\section{EI trabajo y la educación en la seguridad societal}

¿Educarse o trabajar?, es la interrogante que desde comienzos de la década de los años noventa se hizo cotidiana, particularmente después de la argumentación del libro "l fin de la 
Historia y el último hombre ("The End of History and the Last Man") de Francis Fukuyama, cuya idea secundario pero muy relevante, es que el trabajo como se concibe en el marxismo no es el factor económico que determina el progreso en el devenir histórico, sino que es el conocimiento científico como activo intangible expresado en capital social, el que determina el progreso de la humanidad en lo económico y societal.

El capital social como categoría de interés en la investigación, contextualizado en el binomio Educación y Trabajo, se define así [FUKUYAMA, 2001]:

El capital social es una fórmula que promueve la cooperación entre dos o más individuos. Las normas que rigen el capital social, varían desde normas que establecen la reciprocidad entre dos amigos, hasta doctrinas complejas y elaboradamente articuladas como el cristianismo o el confucianismo. La norma de reciprocidad existe en potencia en mis tratos con todas las personas, en las relaciones humanas reales, pero que se fortalece en los vínculos que se establecen a través del capital social. Según esta definición, la confianza, las redes, la sociedad civil y similares que se han asociado con el capital social son epifenómenos, que surgen como resultado del capital social pero no constituyen el capital social en sí mismo.

Se observa entonces que el capital social es producto del esfuerzo de individualidades para producir bienes tangibles o intangibles, partir del trabajo y la educación asumidos como procesos que no están explícitos en la definición de Fukuyama, pero son los que efectivamente en unas relaciones de producción muy sui generis, producen bienes materiales e inmateriales en las sociedades del conocimiento, con una dinámica que exigen de seguridad societal, en un ecosistema con diversos actores, con intereses antagónicos unos, e intereses cooperativos otros, pero que en suma ambos compiten por trabajo y educación. Gramsci integró al trabajo y la educación [MONASTA, 1993], considerando los valores intrínsecos de tipo intelectual y físico, presentes en el trabajo y la educación, como procesos complejos en las relaciones de producción de las sociedades del conocimiento:

En cualquier trabajo físico, aunque sea el más mecánico y degradado, existe un mínimo de calificación técnica, esto es, un mínimo de actividad intelectual creadora, (...) No existe actividad humana de la cual se pueda excluir toda intervención intelectual, no se puede separar el homo faber del homo sapiens.

En la era del homo faber y del homo sapiens en la prospectiva gramsciana; y evidenciando que hoy Fukuyama presenta la antisitesis a lo que esbozó en su famosa obra publicada en 1990, es decir, que el trabajo mancomunado de ese su "último hombre", constituye lo que él ahora 
conceptualiza como capital social, el cual integra al trabajo y la educación, como sistemas y procesos, independientemente de las modalidades que se adopten con la facilitación de las tecnologías disponibles en el ciberespacio.

Con los anteriores referentes teóricos se amplía la óptica de los autores, aunado a lo que se identifica como cuerpo doctrinario en la Constitución de la República Bolivariana, que consagra un sistema educativo que tiene como referentes a los pedagogos Andrés Bello y Simón Rodríguez, al político y estratega Simón Bolívar; cuyos pensamientos en cuanto a educación y trabajo fueron de vanguardia en su tiempo histórico, y aun hoy con los avances de la ciencia y la técnica siguen vigentes. La educación en la República Bolivariana de Venezuela se asume con respeto a la democracia participativa y protagónica, la responsabilidad social, la igualdad entre todos los ciudadanos y ciudadanas sin discriminaciones de ninguna índole, la formación para la independencia, la libertad y la emancipación, la valoración y defensa de la soberanía del pueblo, la formación en una cultura para la paz, la justicia social, el respeto a los derechos humanos, la práctica de la igualdad, justicia social, equidad y la inclusión; la sustentabilidad del desarrollo, el derecho a la igualdad de género, el fortalecimiento de la identidad nacional, la lealtad a la patria e integración latinoamericana y caribeña; y abierta a todas las corrientes de pensamiento científico y filosófico, con equilibrio ideológico.

El sistema educativo venezolano tiene como como valores fundamentales, el respeto a la vida, el amor y la fraternidad, la convivencia armónica en el marco de la solidaridad, la corresponsabilidad, la cooperación, la tolerancia y la valoración del bien común, la valoración social y ética del trabajo, la educación como derecho humano y social, el respeto a la diversidad propia de los diferentes grupos humanos, e igualmente se establece que la educación es pública y social, obligatoria, gratuita, de calidad, de carácter laico, integral, permanente, con pertinencia social, creativa, artística, innovadora, crítica, pluricultural, multiétnica, intercultural, y plurilingüe. La educación en Venezuela es un derecho humano y un deber social, orientado al desarrollo del potencial creativo de cada ser humano en condiciones históricamente determinadas, constituye el eje central en la creación, transmisión y reproducción de las diversas manifestaciones y valores culturales, invenciones, expresiones, representaciones y características propias para apreciar, asumir y transformar la realidad. El Estado venezolano, el cual es democrático, social, de derecho y de justicia; asume la educación como proceso esencial para promover, fortalecer y difundir los valores culturales de la venezolanidad.

El sistema educativo es un conjunto orgánico y estructurado, conformado por subsistemas, niveles y modalidades, de acuerdo con las etapas del desarrollo humano. Se basa en los postulados de unidad, corresponsabilidad, interdependencia y flexibilidad. Integra políticas, planteles, 
servicios y comunidades para garantizar el proceso educativo y la formación permanente de la persona sin distingo de edad, con el respeto a sus capacidades, a la diversidad étnica, lingüística y cultural, atendiendo a las necesidades y potencialidades locales, regionales y nacionales. La Ley Orgánica de Educación de Venezuela establece que el sistema educativo venezolano está integrado por:

1.El subsistema de educación básica, conformado por los niveles de educación inicial, educación primaria y educación media. El nivel de educación inicial comprende las etapas de maternal y preescolar destinadas a la educación de niños y niñas con edades comprendidas entre cero y seis años. El nivel de educación primaria comprende seis años y conduce a la obtención del certificado de educación primaria. En el nivel de educación media tiene una vertiente de educación media general con duración de cinco años, y otro trayecto de educación media técnica con duración de seis años. Ambas opciones conducen a la obtención del título correspondiente para el nivel de educación media.

2. El subsistema de educación universitaria comprende los niveles de pregrado y postgrado universitarios.

3. Las modalidades como variantes educativas para la atención de las personas o grupos societales que por sus características, adaptaciones curriculares de forma permanente o temporal con el fin de responder a las exigencias específicas de la modalidad. La educación especial, la educación de jóvenes, adultos y adultas, la educación en fronteras, la educación rural, la educación para las artes, la educación militar, la educación intercultural y la educación intercultural bilingüe, son las modalidades que se desarrollan actualmente en el sistema educativo.

Es así, que el sub-sistema de educación universitaria, es pertinente para en este artículo ilustrar las relaciones entre trabajo, educación y seguridad societal. La universidad más antigua de Venezuela que nació después de la independencia acaecida el 24 de junio de 1821, es la Universidad Central de Venezuela. En 1827, el Libertador Simón Bolívar como Jefe del Estado aprueba los nuevos Estatutos Republicanos de la Universidad de Caracas, en los cuales se prohibió la discriminación social y racial para ingresar a estudiar a la universidad y para el ejercicio de la docencia universitaria, igualmente y por iniciativa del Libertador, la Universidad de Caracas recibió bienes inmuebles, entre ellos las mejores haciendas de cacao del país, para permitirle a la institución disponer de las rentas suficientes para su funcionamiento y crecimiento con autonomía en lo financiero y escolástico. 
En este siglo XXI, justo a partir del año 2000, se refunda Venezuela como Estado-nación con la Constitución de la República Bolivariana de Venezuela, aprobada en refrendo popular el 15 de diciembre de 1999. La carta magna de Venezuela, honra el principio de la autonomía universitaria, que el Libertador Simón Bolívar a la Universidad de Caracas que fundó en 1827, con las definiciones y mandato que se transcribe a continuación del Artículo 109 [CONSTITUCIÓN DE LA REPÚBLICA BOLIVARIANA DE VENEZUELA, 1999]:

El Estado reconocerá la autonomía universitaria como principio y jerarquía que permite a los profesores, profesoras, estudiantes, estudiantas, egresados y egresadas de su comunidad dedicarse a la búsqueda del conocimiento a través de la investigación científica, humanística y tecnológica, para beneficio espiritual y material de la Nación. Las universidades autónomas se darán sus normas de gobierno, funcionamiento y la administración eficiente de su patrimonio bajo el control y vigilancia que a tales efectos establezca la ley. Se consagra la autonomía universitaria para planificar, organizar, elaborar y actualizar los programas de investigación, docencia y extensión. Se establece la inviolabilidad del recinto universitario. Las universidades nacionales experimentales alcanzarán su autonomía de conformidad con la ley.

Desde el Estatuto de 1827, las universidades venezolanas han evolucionado, pero con la rémora de las costumbres de la antigua institucionalidad universitaria al servicio de la monarquía de los Reyes de Castilla, con el arraigo de una cultura universitaria que asume la autonomía como una condición que le da el privilegio de enclaustrarse para usufructuar el poder y privilegios [MORGENTHAU, 1963], y desde la territorialidad del campus universitario limitar las relaciones saludables de la comunidades al interno y con su entorno, como cuerpo societal que con garantía de la seguridad debería ser la universidad. Ante esas aberraciones, la academia venezolana esta llamada a debatir, de forma plural, y por ejemplo, incorporar al debate como referente paradigmático, al modelo de cuádruple hélice, pues este contempla a la sociedad civil, que a juicio de los autores podría contribuir a ampliar la visión de las universidades venezolanas en cuanto al concepto de autonomía universitaria. En un paradigma emergente de autonomía universitaria, la seguridad societal será de importancia capital, cuando consideramos los conflictos potenciales que el claustro enfrentará con la apertura al ciberespacio para elevar la calidad educativa universitaria; las universidades nacionales venezolanas se verán obligadas de facto a salir de su enclaustramiento, para efectivamente garantizar la concreción de las políticas de movilidad en la territorialidad y en espacios públicos virtuales.

Las universidades nacionales de Venezuela, deberían considerar en sus análisis de entorno al proyecto MIME, financiado por la European Commission's Seventh Framework Programme 
de Unión Europe [MOBILITY AND INCLUSION IN MULTILINGUAL EUROPE, 2014]. El MIME mediante un innovador enfoque interdisciplinario, generará un cuerpo organizado de propuestas relevantes como insumo para la formulación de políticas públicas, identificando las políticas y estrategias lingüísticas que mejor combinen "movilidad" e "inclusión”. Los diversos conceptos y métodos se integran en un marco lógico y analítico, diseñado para garantizar su integración educativa en Europa y evitar la conflictividad societal en los sistemas educativos de los países de la Unión Europea.

En relación a la escalada de la Guerra no Convencional que sufre la población venezolana de parte de Estados Unidos, cuando a comienzos de marzo de 2015, el Presidente Barack Obama señaló a la República Bolivariana de Venezuela como una "amenaza inusual y extraordinaria a la seguridad nacional; y a la política exterior de Estados Unidos" [OBAMA, 2015]; las universidades nacionales venezolanas se han debilitado por esa agresión de Estados Unidos a Venezuela, al punto, que gran cantidad de profesores han renunciado a sus empleos, y una cantidad de estudiantes se han retirado de las carreras profesionales que estaban cursando. Aunado a ello, la administración actual del Estado venezolano, ha tenido algunas vulnerabilidades desde el año 2013, en relación al diseño y desarrollo de políticas para el sub-sistema de educación universitaria, que hubiesen evitado que prácticamente el salario real de los docentes universitarios en la actualidad sea inexistente, al igual que no existen para los estudiantes ningún incentivo como podrían ser las becas o créditos educativos para sufragar sus gastos de alimentación, transporte, recreación, libros, material didáctico, acceso a internet, entre algunos otros.

En la realidad concreta, no existe remuneración del trabajo de los profesores, empleados y personal obrero al servicio de las universidades nacionales venezolanas en la actualidad [PROFESSORS FLEE AND HIGHER EDUCATION SUFFERS IN VENEZUELA, 2015]; al igual que la educación de los estudiantes universitarios se limita grandemente porque renuncian profesores de una larga experiencia académica y científica; poniendo en altísimo riesgo al sistema de educación venezolana y consecuentemente crea un problema y una amenaza latente a la seguridad societal en las instituciones de educación universitaria venezolanas.

Ante esta coyuntura, la mayoría de las autoridades rectorales de universidades nacionales con la autonomía como legado del Estatuto republicano promulgado por el Libertador Simón Bolívar en 1827, se aprecia que ellas no han diseñado e implementado un Sistema Gerencial para la Continuidad de las actividades en las Universidades (Estándar ISO 22301:2012 "Seguridad Societal"), en el fragor de la Guerra No Convencional de Estados Unidos contra la población venezolana, para defender corresponsablemente a la República Bolivariana de Venezuela. En lugar de ello, la mayoría de las autoridades rectorales se han amparado en la figura del claustro 
profesoral, queriéndose hacerse vitalicios en los cargos, oponiéndose también a que se realicen elecciones libres y democráticas para elegir nuevas autoridades. Se oponen visceralmente a que las comunidades universitarias ejerzan sus derechos políticos, civiles y educativos en el contexto de lo que consagra la legislación en materia educativa [LEY ORGÁNICA DE EDUCACIÓN, 2009]:

En aquellas instituciones de educación universitaria que les sea aplicable, el principio de autonomía reconocido por el Estado se materializa mediante el ejercicio de la libertad intelectual, la actividad teórico-práctica y la investigación científica, humanística y tecnológica, con el fin de crear y desarrollar el conocimiento y los valores culturales. La autonomía se ejercerá mediante las siguientes funciones: 1. Establecer sus estructuras de carácter flexible, democrático, participativo y eficiente, para dictar sus normas de gobierno y sus reglas internas de acuerdo con lo establecido en la Constitución de la República y la ley; 2. Planificar, crear, organizar y realizar los programas de formación, creación intelectual e interacción con las comunidades, en atención a las áreas estratégicas de acuerdo con el Plan de Desarrollo Económico y Social de la Nación, las potencialidades existentes en el país, las necesidades prioritarias, el logro de la soberanía científica y tecnológica y el pleno desarrollo de los seres humanos; 3 . Elegir y nombrar sus autoridades con base en la democracia participativa, protagónica y de mandato revocable, para el ejercicio pleno y en igualdad de condiciones de los derechos políticos de los y las integrantes de la comunidad universitaria, profesores y profesoras, estudiantes, personal administrativo, personal obrero y, los egresados y las egresadas de acuerdo al Reglamento. Se elegirá un consejo contralor conformado por los y las integrantes de la comunidad universitaria; y 4. Administrar su patrimonio con austeridad, justa distribución, transparencia, honestidad y rendición de cuentas, bajo el control y vigilancia interna por parte del consejo contralor, y externa por parte del Estado. El principio de autonomía se ejercerá respetando los derechos consagrados a los ciudadanos y ciudadanas en la Constitución de la República, sin menoscabo de lo que establezca la ley en lo relativo al control y vigilancia del Estado, para garantizar el uso eficiente del patrimonio de las instituciones del subsistema de educación universitaria. Es responsabilidad de todos y todas, los y las integrantes del subsistema, la rendición de cuentas periódicas al Estado y a la sociedad sobre el uso de los recursos, así como la oportuna información en torno a la cuantía, pertinencia y calidad de los productos de sus labores.

Como consecuencia de que en 10 universidades venezolanas con carácter de autonomía, sus autoridades han permanecido por más de diez años en los cargos sin un Plan estratégico de 
Desarrollo Institucional, siendo un factor importante de incitación a las protestas violentas contra el gobierno en 2014 y 2017 por grupos hegemónicos apoyados por la prolongada permanencia de estas autoridades no renovadas. Ante un recurso de interpretación de la Universidad Central de Venezuela, el Tribunal Supremo de Justicia de Venezuela en agosto de 2019 dictó una sentencia, para garantizar el derecho al voto de la comunidad universitaria e indirectamente contribuir con la seguridad societal del sub-sistema de educación universitaria [TRIBUNAL SUPREMO ORDENA A LA UNIVERSIDAD CENTRAL DE VENEZUELA ELEGIR NUEVAS AUTORIDADES, 2019].

Para resolver el dilema operativo del cambio del sistema de elección, los autores de éste artículo estudiaron los aportes de un grupo de docentes e investigadores que hacen vida en la Universidad Simón Bolívar, cuyo constructo para el esquema de elección de las autoridades rectorales en 10 universidades venezolanas que ejercen autonomía, contempla el Sufragio o Votación Electoral por cada sector de los cinco sectores societales, que conforman la comunidad universitaria en conformidad con la Ley Orgánica de Educación en Venezuela: personal Académico, personal Estudiantil, personal de Egresados, personal Administrativo y personal de Servicios. Cada sector en su totalidad representa en valor un solo voto electoral para la elección de las autoridades rectorales, a ser ganado por mayoría de votación de los electores propios con membresía en el sector correspondiente. Para ser elegido como autoridad rectoral, es necesario que el candidato obtenga 3 de 5 votos electorales que generan la totalidad de los sectores.

En cada uno de los cinco sectores se votará con una boleta electoral donde el elector puede seleccionar el lugar de preferencia de cada candidato: primero y segundo, por ejemplo. Obtendrá el Voto Electoral del sector correspondiente, el candidato que obtenga la mitad más 1 de los votos válidos emitidos, es decir por mayoría absoluta. Si no hubiese un ganador claro en un sector, de forma iterativa se selecciona para descarte en el proceso de votación al candidato con menor votación de ese sector y se procede a repartir los votos de acuerdo a la mayor preferencia vigente entre los candidatos remanentes, hasta que haya un ganador con mayoría absoluta.

La sentencia del Tribunal Supremo de Justicia adoptó el esquema de elección por mayoría en los cinco sectores, pero no el segundo sobre el Voto Preferencial transferible, dado que la segunda vuelta electoral entre los dos candidatos más votados no fue objeto de cuestionamiento en la demanda de interpretación interpuesta ante el TSJ; por lo cual el máximo tribunal de la República Bolivariana de Venezuela decidió adaptar este aspecto especificado en la Ley de Universidades vigente, incorporando una segunda vuelta entre los candidatos que hubieran obtenido más sectores y votos, en el caso que ninguno de ellos alcanzase al menos 3 de los 5 sectores y no obtenía la mitad más uno de los votos válidos totales. Además, esta sentencia del

P/U $a$ i $S$ Salvador, v. 4, n. 3, p. 09-35, set./dez. 2019 
TSJ, libera de la tutela de los Consejos Universitarios a las Comisiones Electorales, y les faculta y responsabiliza de que la elección se lleve a término, expandiendo aún más el concepto de autonomía universitaria.

El esquema que decidió el TSJ para elegir a las autoridades rectorales en Venezuela, es una variante del que se conoce como Voto Preferencial Transferible con vuelta instantánea, el cual es usado para elegir al Presidente de Irlanda, a los Representantes en las elecciones parlamentarias de Australia, a los Consejeros en las alcaldías de Londres (Reino Unido) y San Francisco (Estados Unidos), y en las elecciones internas de los partidos Laborista (Reino Unido) y Liberal (Canadá). En resumen, para las próximas elecciones de las autoridades rectorales universitarias en Venezuela, el Estado venezolano ha actuado, respetando los méritos académicos en el marco de la autonomía universitaria; al igual que los principios y valores de democracia participativa, corresponsabilidad societal, responsabilidad social; justicia, equidad, libertad, igualdad y paz; y con reconocimiento pleno del ejercicio de los derechos civiles y políticos, el derecho a la educación y el trabajo, con una orientación teleológica a la seguridad societal.

\section{Reflexión conclusiva}

La seguridad societal es un paradigma emergente en algunos países del mundo, paticularmente los de Latinoamérica y el Caribe, y ante todo su desarrollo ha estado impulsado mayormente desde actores económicos fundamentales, como son las organizaciones empresariales. Para la consolidación de la seguridad societal, son imprescindibles el trabajo y la educación. En consecuencia, dependiendo de sus grados de fortaleza, el trabajo y la educación pueden crear o no vulnerabilidades, peligros y amenazas a la seguridad societal, que inciden sobre el riesgo calculado y apreciado en las empresas e instituciones de educación.

Tradicionalmente en los estudios sobre seguridad seguridad societal, se ha identificado que las migraciones internas e externas de grupos sociales en los Estados-nación y en el escenario internacional, son fuentes de amenazas, peligros y vulnerabilidades. Es por ello que cuando se generan crisis económicas o conflictos de diversa naturaleza e intensidad, como Guerras no Convencionales, se producen flujos de grupos humanos, perdiéndose el sentido de pertenencia y cohesión societal. En el caso de las universidades, se debería elevar la conciencia en la necesidad de preservar la seguridad societal como valor superior, y diseñar un Sistema Gerencial para la Continuidad de los Procesos Universitarios que minimicen la renuncia de profesores, personal administrativo, personal de servicios y el abandono de los programas formativos por parte de los estudiantes; con participación activa de todos los miembros de la comunidad, independiente de su 
jerarquía, credo religión, etnicidad e ideologías; aplicado y adaptándose al modelo de Cuádruple Hélice para la innovación.

Como un factor que fortalecería la seguridad societal de las universidades autónomas venezolanas, se identificó a la sentencia dispositiva del Tribunal Supremo de Justicia venezolano, en la cual se ordena que en un plazo máximo de 6 meses a las universidades con carácter de autonomía, elegir a sus autoridades rectorales para la renovación de la mayoría de las autoridades que han permanecido en funciones por más de diez años. En conformidad con la Ley Orgánica de Educación de Venezuela, las elecciones deberán respetar los valores y méritos de quienes opten como candidatos, al igual permitir la inclusión de todos los sectores de forma plural y respetando los principios de la Democracia Participativa; a los fines de que quienes sean electos tengan legitimidad y devuelvan la gobernanza a las instituciones universitarias en un clima de Paz social y equilibrio ideológico en el ambiente natural en la perspectiva del paradigma emergente de Elías Carayannis y David Campbell o en un ambiente artificial como lo es el ciberespacio [HOLDER, 2018].

Con las elecciones de autoridades rectorales que exige el Tribunal Supremo de Justicia de Venezuela para las universidades autonómicas, además se restablecerá la continuidad de la institucionalidad en el contexto del estándar ISO 22301:2012, impedida por la fosilización de las autoridades en sus cargos por más de una década. Se espera también se desarrolle de manera amplia y justa la autonomía interna para elegir los cargos, no sujeta a la línea de mando de las autoridades con el período vencido. Las Comisiones Electorales al interno de las universidades autonómicas venezolanas, harán uso de esta nueva independencia y libertad, para hacer cumplir ajustadas a derecho, la sentencia del máximo tribunal de la República Bolivariana de Venezuela. Las universidades autonómicas venezolanas tienen más de diez años sin elecciones, sin Planes Estratégicos de Desarrollo Institucional, sin actualización de los Planes de Estudios y el Currículo; han prácticamente paralizado al sub-sector de educación universitaria de Venezuela, y lo más grave es que han vulnerado al trabajo y a la educación como procesos fundamentales para el logro de los fines del Estado venezolano. En esas materias que tienen pendiente aprobar las universidades autonómicas venezolanas, deberán perentoriamente en no más de seis meses, colocarse también a la vanguardia de la defensa integral de la educación y el trabajo, como derechos sociales y humanos, a los fines de garantizar la Seguridad Societal en los centros de enseñanza en general [GAIRÍN SALLÁN, 2012]. 


\section{REFERENNCIAS}

AALTOLA, M. et Al. "Societal Security in the Baltic Sea region: Expertise Mapping and Raising Policy Relevance". Riga: Publicación del "Latvian Institute of International Affairs", 2018.

BUZAN, Barry. People, States, and Fear: An Agenda for International Security Studies in the Post-Cold War Era. Sussex: Wheatsheaf, 1983.

CARAYANNIS, Elias y CAMPBELL, David. Mode 3 and Quadruple Helix: toward a 21st century fractal innovation ecosystem. International Journal of Technology Management, Vol. 46, $\mathrm{N}^{\circ} 3 / 4$, p. 201-234, 2009.

CENTRO DE INVESTIGACIÓN DE PROTECCIÓN URBANA Y SEGURIDAD SOCIETAL (URBSEC). 2019. Disponible en: http://www.urbsec.se/ . Consulta: 10SEP2019.

CENTRO DE VIENA PARA LA SEGURIDAD SOCIETAL (VICESSE). 2019. Disponible en: https://www.vicesse.eu/source . Consulta: 10SEP2019.

CONSTITUCIÓN DE LA REPÚBLICA BOLIVARIANA DE VENEZUELA (CRBV). 1999. Disponible en: el 10SEP2016 en: https://www.oas.org/juridico/mla/sp/ven/sp_ven-int-const.html . Consulta: 10SEP2016.

DE ESPONA, Rafael. Seguridad del báltico y vínculo euroatlántico: 25 años de la restauración de la independencia de Lituania. Madrid: Instituto Español de Estudios Estratégicos, 2015.

ESTECCHE DE FERNÁNDEZ, Elianne. El derecho societario venezolano en el siglo XXI y las sociedades agrarias. Revista Derecho y Reforma Agraria Ambiente y Sociedad de la Universidad de los Andes, N 40, p. 43-62, Dic. 2014.

FUKUYAMA, Francis. Social capital, civil society and development. Third World Quarterly, Vol. 22, No 1, p. $7-20,2001$.

GAIRÍN SALLÁN, Joaquin et Al. La seguridad integral en los centros de enseñanza obligatoria de España. Barcelona: C FUNDACIÓN MAPFRE (Instituto de Prevención, Salud y Medio Ambiente) y UNIVERSIDAD AUTÓNOMA DE BARCELONA (Grupo de Investigación EDURISC del Centre de recerca en Governança del Risc y al Equipo de Desarrollo Organizacional), 2012.

GONZÁLEZ, Franklin. Qué es la seguritización. 2016. Disponible en: https://www.aporrea.org/ actualidad/a225628.html. Consulta: 12ABR2016. 
HOLDER, Luis. La ideología en la conceptualización de la Seguridad de la Nación. Revista COLUMNATA de la Universidad Militar Bolivariana de Venezuela, Nº 6, p. 165-180, Jun. 2014.

HOLDER, Luis y CÁRDENAS, Julio. Strategic Concepts for Training in Integrated Defence of Peace and Security in Cyberspace (Capítulo 6 del libro "Risks, Violence, Security and Peace in Latin America. 40 Years of the Latin American Council of Peace Research"). Berlín: International Publishing (Edición impresa: ISBN 978-3-319-73807-9 y Edición electrónica ISBN 978-3319-73808-6), 2018.

LEY ORGÁNICA DE EDUCACIÓN. 2009. Disponible en: hhttp://www.siteal.iipe.unesco.org/ bdnp/583/ley-organica-educacion-go-ndeg-5929-ley-organica-educacion. Consulta: 10SEP2016.

MALDONADO MICHELENA, Victor. “Las Naciones y su Defensa Integral”. Caracas: Editorial Drusa, 1962.

MESSINA, Graciela et Al. "Educación y Trabajo: Lecciones desde la práctica innovadora en América Latina”. Santiago de Chile: Oficina Regional de Educación de la UNESCO para América Latina y el Caribe (OREALC/UNESCO), 2008.

MIME (Mobility and Inclusion in Multilingual Europe). 2019. Disponible en: https://www. mime-project.org. Consulta: 10SEP2019.

MOLLER, Bjørn. Seguridad Nacional, Societal y Humana: El marco general y el caso de los Balcanes. Encuentro Internacional de Directores de instituciones de capacitación e investigación. Paris: UNESCO-FASOC, p. 3-28. Oct. 2000.

MONASTA, Attilio. Antono Gramsci. Revista de Educación comparada, Vol. XXIII, N 3-4, París: UNESCO, p. 633-649, 1993.

MORGENTHAU, Hans. La Lucha por el Poder y por la Paz. Buenos Aires: Editorial Sudamericana, 1963.

OBAMA, Barack. 2015. Disponible en: https://obamawhitehouse.archives.gov/the-press-office/2015/03/09/fact-sheet-venezuela-executive-order. Consulta: 10SEP2019.

ORGANIZACIÓN INTERNACIONAL DE NORMALIZACIÓN (ISO). 2019. Disponible en: https://www.iso.org. Consulta: 11SEPP2019:

PROFESSORS FLEE AND HIGHER EDUCATION SUFFERS IN VENEZUELA. 2015. Disponible en: https:/www.apnews.com/e99dc9ca55144331851150a67eaa4862. Consulta: 
10SEP2019.

RODRÍGUEZ, Dario y ARNOLD, Marcelo. "Sociedad y Teoría de Sistemas: Elementos para la comprensión de la teoría de Niklas Luhmann”. Santiago de Chile: Editorial Universitaria, 1990.

SCHÜTZ, Florian et Al. "Co-shaping the Future in Quadruple Helix Innovation Systems:

Uncovering Public Preferences toward Participatory Research and Innovation". The Journal of Design, Economics, and Innovation, Volume 5, $\mathrm{N}^{\circ}$ 2, p. 128-146, 2019.

SECRETARÍA DE SEGURIDAD MULTIDIMENSIONAL DE LA OEA. Disponible en: http:// www.oas.org/es/temas/seguridad_multidimensional.asp. Consulta: 10SEP2019.

The Triple Helix: A Laboratory for Knowledge Based Economic Development. 1995. Disponible en: https://papers.ssrn.com/sol3/papers.cfm?abstract_id=2480085. Consulta: 11SEP2019.

UNIÓN INTERNACIONAL DE TELECOMUNICACIONES (ITU). 2019. Disponible en: https://www.itu.org. Consulta: 11SEP2019.

TRIBUNAL SUPREMO ORDENA A LA UNIVERSIDAD CENTRAL DE VENEZUELA ELEGIR NUEVAS AUTORIDADES. 2019. Disponible en: http://spanish.xinhuanet.com/2019-08/28/c_138344809.htm. Consulta: 11SEP2019.

Enviado em: 12 de setembro de 2019.

Apreciado em: 30 de setembro de 2019.

Inserido em: 10 de dezembro de 2019 